\title{
Effects of a Wind-Driven Gyre on Thermohaline Circulation Variability
}

\author{
Claudia Pasquero \\ Istituto di Scienze dell'Atmosfera e del Clima, CNR, Turin, Italy \\ ELI TZIPERMAN \\ Environmental Sciences, Weizmann Institute, Rehovot, Israel
}

(Manuscript received 19 February 2002, in final form 21 October 2003)

\author{
ABSTRACT
}

\begin{abstract}
A simplified model is used to study the possible effects of the horizontal upper-ocean wind-driven circulation (WDC) on the variability of the overturning meridional circulation driven by buoyancy fluxes. It is found that the added interaction with the WDC adds interesting new classes of variability. First, self-sustained variability of the thermohaline circulation (THC) becomes possible, on time scales of interdecades to a few centuries. Furthermore, these oscillations may be either small amplitude or large amplitude and either periodic or chaotic, depending on the amplitude of the freshwater forcing and on the strength of the WDC. Even a relatively weak WDC changes the well-known stability properties of the THC that are seen in numerous models of the THC alone. The variability modes found here may account for similar modes of variability observed in GCM studies.
\end{abstract}

\section{Introduction}

North Atlantic sea surface temperature (SST) variability can have a significant impact on the climate system (Dickson et al. 1996; Manabe and Stouffer 1999; Moron et al. 1998). The variability up to interannual time scales is largely due to atmospheric stochastic forcing; at longer time scales the internal ocean dynamics play a role in generating SST anomalies (Bjerknes 1964). The dominant internal ocean variability modes on the large scale are due to the thermohaline circulation (THC) and the wind-driven circulation (WDC). Observations indeed link interdecadal sea surface temperature variability to the overturning meridional circulation variability (Kushnir 1994); the variability of the horizontal wind-driven circulation plays a role in observed variability on time scales of about a year and longer (Dijkstra 2000).

There have been numerous studies of the separate variability of the WDC and the THC, using a hierarchy of models from simple box models to full ocean and coupled ocean-atmosphere general circulation models [OGCMs and CGCMs; see Dijkstra (2000) for a comprehensive review]. However, there has been much less work on coupled variability modes of the WDC and the THC. Those studies that do address such coupled variability of the WDC and THC are mostly based on GCM

Corresponding author address: Claudia Pasquero, ISAC CNR, Corso Fiume 4, 10133 Turin, Italy.

E-mail: claudia@icg.to.infn.it integrations (Delworth and Mann 2000; Delworth et al. 1993; Eden and Jung 2001; Hall and Stouffer 2001; Latif 1998; Latif and Barnett 1994), which makes it difficult to explore basic dynamical issues that are more accessible using simplified models.

The objective of the present work is to use a relatively simple model of the WDC and THC to explore mechanisms of interaction between the two. In fact, we are mostly interested here in understanding how the existence of a WDC affects the variability modes of the THC.

There are two general classes of interaction between the WDC and the THC that one can envision. First, the WDC may advect salinity anomalies around the gyre, and at some stage they may reach the area of deep-water formation and enhance or shut off the overturning circulation (Delworth et al. 1993; Weaver and Sarachik 1991; Winton and Sarachik 1993; Yin and Sarachik 1995). Second, a THC variability may cause SST variability, leading to changes in the atmospheric circulation and therefore to changes to the wind stress, which can affect the WDC (Delworth and Mann 2000; Dong and Sutton 2001; Eden and Jung 2001; Hall and Stouffer 2001; Latif and Barnett 1996; Marshall et al. 2001). We are interested here only in the first class of interactions, in which the WDC is taken as constant in time, ignoring the effects of the SST on the wind as well as various stochastic forcings of the WDC (Frankignoul et al. 1997), and our model formulation follows this assumption. The choice is motivated by the desire to consider the simplest possible model of the effects of a wind- 
driven gyre on the thermohaline circulation. As will become clear shortly, this one-way interaction between the WDC and THC already introduces many interesting new results that are worthy of their study here. In a subsequent work, a simple parameterization of the feedbacks of the SST on the wind stress and hence the twoway interaction between the THC and WDC will be studied.

It is our hope that the work presented here may shed some light on the findings of GCMs that have seen hints of interaction between the THC and WDC. In addition, the results of the present study extend in an interesting way our understanding of THC variability as follows. Several classes of THC variability have been proposed over the past few years, and most of these were studied using a hierarchy of models from GCMs to toy models. Damped linear THC oscillations excited by stochastic atmospheric forcing were studied, for example, by Griffies and Tziperman (1995) using a simple box model and interpreting the coupled GCM study of Delworth et al. (1993). Self-sustained small-amplitude THC variability was found by Chen and Ghil (1995) in a fairly complex model. Rivin and Tziperman (1997) used a simple box model to show that it is very difficult to get self-sustained THC oscillations in a model of the THC only, where the circulation linearly depends on meridional density gradients only, without making some artificial assumptions. We show here that, by merely adding the simplest possible representation of the WDC, one obtains a rich self-sustained THC variability.

Larger-amplitude THC oscillations caused by jumps between different equilibria induced by stochastic forcing was studied in idealized models by Cessi (1994) and in an OGCM by Weaver and Hughes (1994). Self-sustained large-amplitude THC oscillations that involved switches from positive to negative THC cells were found in the GCM study of Weaver et al. (1991). Again, this rich behavior of large-amplitude THC variability is not reproduced in idealized toy models of the meridional overturning circulation, and one wonders what is the missing element that is required to obtain such interesting behavior. We show here that, by including the WDC effects, we can obtain self-sustained large-amplitude THC variability, periodic and even chaotic switches from thermally dominant to salinity-dominant $\mathrm{THC}$, and more.

We refer in several places below to the difficulty of obtaining self-sustained THC variability in simple THC models. There are basically two classes of oscillatory THC variability. One is related to the loop oscillation of Welander (1986) in which the large-scale temperature and salinity gradients drive the oscillation. This class often results in damped THC variability that in most simple models needs some external stochastic atmospheric forcing to be excited (Griffies and Tziperman 1995). This class is also the one for which we show here that self-sustained THC variability becomes possible with the addition of the WDC. The other class is related to the local behavior of convection in watermassformation areas (flip-flop model; Welander 1982). This variability, which is unrelated to our main theme here, is due to the nonlinear dependence of the convective mixing on the vertical density gradient or is due to the nonlinear equation of state. This second class of thermohaline variability has different characteristics when forced stochastically (Cessi 1996) and was observed in GCMs (e.g., Pierce et al. 1995; Winton and Sarachik 1993).

Present-day THC variability is most likely of a fairly small amplitude (5\%-10\% of mean value) so that it is most likely stable linear dynamics, excited by stochastic forcing. It is therefore worth noting that some of the parameter regimes explored in this work may not be relevant for present-day climate variability because they involve large-amplitude THC variability. We feel that it is still useful to carry some forcing parameters to extreme values that explore different regimes of THC dynamics so as to gain some insight into possible variability modes in the presence of both the THC and WDC. Such an understanding is also useful in putting variability modes that are more realistic for present-day climate into perspective.

The following sections introduce the model used here (section 2) and then briefly analyze the model stability behavior in the case of no wind-driven circulation (section 3) and in the presence of both WDC and THC (section 4), where time-dependent solutions (self-sustained oscillations and chaotic behavior) are found and analyzed. We conclude in section 5 .

\section{The model}

To study the interaction of the WDC and THC in the simplest possible circumstances, we represent a onehemisphere ocean by two boxes that correspond to polar and midlatitude deep waters, plus an annulus that represents the surface WDC (Fig. 1). The circulation in this model is a superposition of two components. First, there is a WDC component that circulates anticyclonically at a constant angular velocity $\Omega$ in the annulus and is of a specified transport by some implicit wind forcing. The WDC is fixed in time, does not depend on the ocean density field, and neglects any coupled ocean-atmosphere feedbacks from the ocean temperature to the wind stress that may affect the WDC. Second, the THC circulates meridionally through the surface layer and then through the deep boxes, and its amplitude depends on the meridional density gradients, similarly to standard THC box models (Stommel 1961). As the THC leaves the deep boxes to join the surface annulus, its water transport is divided between the two branches of the annulus. For a poleward surface flow of the THC (thermally dominant) the velocity of the water in the surface loop is consequently increased in the western part of the loop, where wind-driven and buoyancy-driven transports are both directed northward, and is reduced 


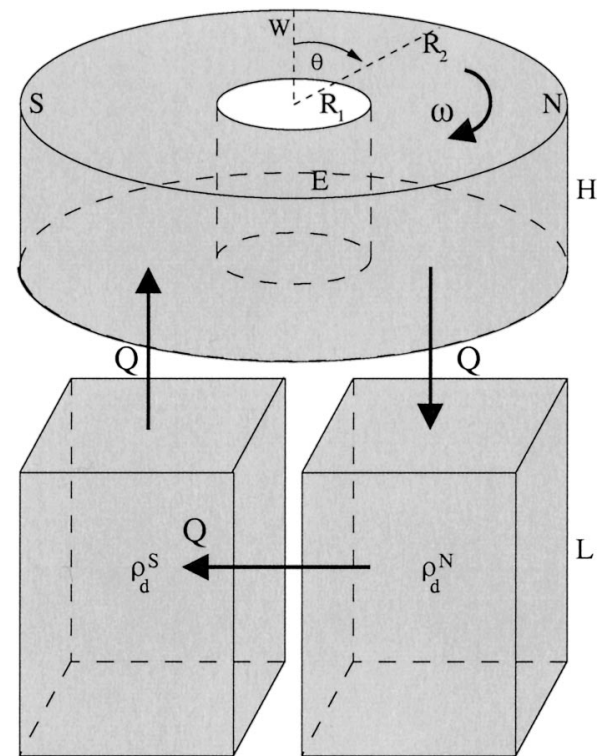

FIG. 1. Schematic of the model geometry: the upper-ocean winddriven circulation is represented by an annulus, and the deeper belowthermocline water is represented by polar and midlatitude boxes.

in the eastern part, where the wind-driven circulation is directed southward and the buoyancy-driven flow is directed northward.

The temperature and salinity fields in the annulus are governed by one-dimensional advection-diffusion partial differential equations, and the temperature and salinity of the deeper boxes are governed by the usual box-model ODEs. At the surface, mixed boundary conditions (Bryan 1986) are imposed: the SST is restored to a fixed profile, and a freshwater (FW) flux that is constant in time is applied. The restoring temperature profile and the FW flux are latitude dependent to simulate crudely the appropriate atmospheric forcing.

The following sections provide a fuller description of the model equations. The reader is advised to proceed on first reading from here directly to the description of the results in section 3 .

\section{a. Surface circulation}

The wind-driven circulation in the layer above the thermocline is represented here as a gyre within an annulus whose depth is $H$, internal radius is $R_{1}$, and external radius is $R_{2}$ (see Fig. 1). The azimuthal coordinate $\theta$ varies in the range $[0,2 \pi]$, where $\theta=0$ defines the west direction and is positive clockwise. The water flux through the bottom of the annulus from the buoyancydriven thermohaline circulation that carries water into and from the deep boxes is given by $F_{W}(\theta)$. The upwelling from the midlatitude box into the annulus is assumed to occur over a wide area, and the sinking from the annulus into the polar box is assumed to occur over a narrow range of $\theta$. When no overturning circulation is present, water in the annulus moves clockwise with a constant uniform angular velocity $\Omega$ that represents the wind-driven gyre circulation. Feedbacks from the SST to the wind stress and WDC are neglected. When an overturning circulation is present, it is added to the surface-layer velocity as explained above. The angular velocity $\omega$ of the water in the loop then becomes dependent on $\theta$, as shown in the following.

The continuity equation in cylindrical coordinates, with the assumption of no motion along the radial direction $r$, is

$$
\frac{1}{r} \frac{\partial v}{\partial \theta}+\frac{\partial w}{\partial z}=0
$$

where $v$ and $w$ are the fluid velocity components in the tangential and vertical directions and $z$ is water depth. Integrating (1) in the vertical direction and using the boundary condition $w(z=H)=-F_{W}(\theta)$ and $w(z=0)$ $=0$, we find

$$
\frac{\partial \omega}{\partial \theta}=\frac{1}{H} F_{W},
$$

with the notation $\omega \equiv v / r$. Next, the water flux $F_{W}(\theta)$ between the surface and deep boxes per unit area of the bottom of the annulus is specified to be

$$
\begin{aligned}
F_{W}(\theta) & =\frac{2 Q}{R_{2}^{2}-R_{1}^{2}} f(\theta) \\
& =\frac{2 Q}{R_{2}^{2}-R_{1}^{2}}\left[-\frac{e^{-(\theta-\pi / 2)^{2} / 2 \sigma_{N}^{2}}}{\sqrt{2 \pi \sigma_{N}^{2}}}+\frac{e^{-(\theta-3 \pi / 2)^{2} / 2 \sigma_{S}^{2}}}{\sqrt{2 \pi \sigma_{S}^{2}}}\right],
\end{aligned}
$$

where $Q$ is the total water flux (in Sverdrup; $1 \mathrm{~Sv} \equiv$ $10^{6} \mathrm{~m}^{3} \mathrm{~s}^{-1}$ ) driven by the thermohaline circulation. The midlatitude upwelling and the polar sinking are represented by two fluxes whose intensities have Gaussian distribution centered around the south $\left(\theta_{S}=3 \pi / 2\right)$ and north $\left(\theta_{N}=\pi / 2\right)$ points, respectively. The variances $\sigma_{S}$ and $\sigma_{N}$ are chosen such that the exchange with the deep ocean is widely distributed in $\theta$ in the upwelling area and narrowly distributed in the downwelling area. The choice of a different reasonable form for the distribution of the water flux $F_{W}(\theta)$ from and to the lower layer does not lead to qualitatively different results. The water flux profile is shown in Fig. 2a for $\sigma_{S}=0.5$ and $\sigma_{N}=0.1$.

Integration of (2), imposing $w\left(\theta_{N}\right)=w\left(\theta_{S}\right)=\Omega$, leads to

$$
\begin{aligned}
\omega= & \Omega+\frac{Q}{\left(R_{2}^{2}-R_{1}^{2}\right) H} \\
& \times\left[-\operatorname{erf}\left(\frac{\theta-\pi / 2}{\sqrt{2 \sigma_{N}^{2}}}\right)+\operatorname{erf}\left(\frac{\theta-3 \pi / 2}{\sqrt{2 \sigma_{S}^{2}}}\right)+1\right],
\end{aligned}
$$

where the constant $\Omega$ represents the clockwise winddriven circulation and the last term represents the thermohaline component of the circulation, directed northward along the two branches of the loop. The ther- 


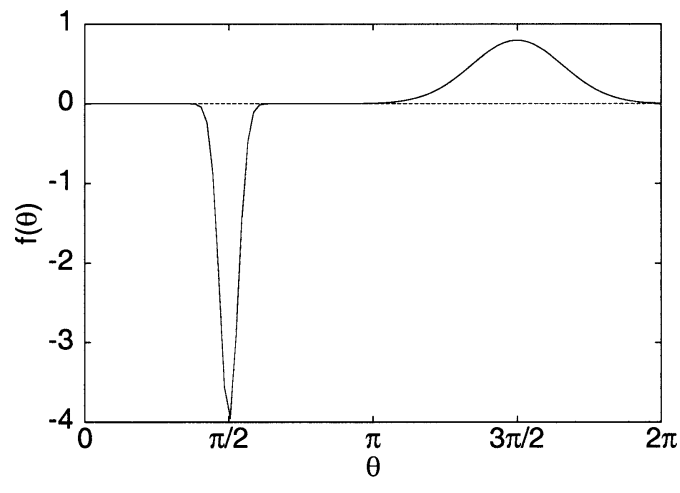

(a)

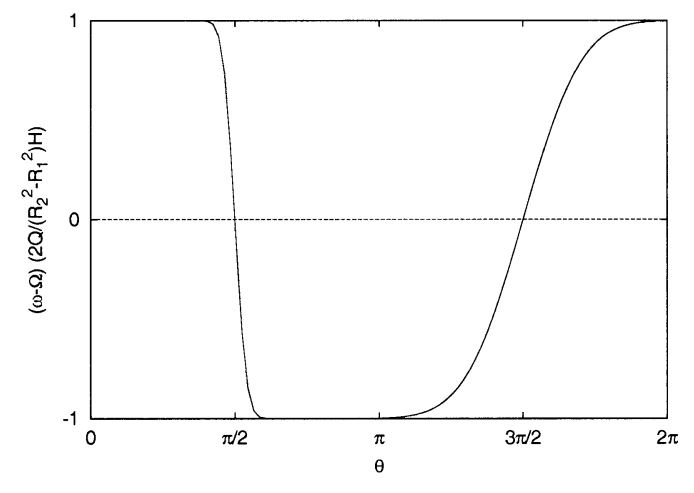

(b)

FIG. 2. (a) The profile of $F_{W}(\theta)$, the water flux between the annulus and the deep boxes, as defined in (3), with $\sigma_{N}$ $=0.1$ and $\sigma_{S}=0.5$. Note the narrow downwelling in the polar region, $0<\theta<\pi$, and broad upwelling in the equatorial region, $\pi<\theta<2 \pi$. (b) The corresponding anomalous (with respect to $\Omega$ ) angular velocity profile in the gyre from (4).

mohaline component of the angular velocity profile is shown in Fig. $2 b$.

Consider next the equation for the salinity $S_{s}$ in the surface annulus. Evaporation and precipitation processes are parameterized as a specified FW flux, $F_{s}=$ $-F_{s}^{0} \sin \theta$, that represents a net evaporation in the equatorial region and a net precipitation in the polar region. The salinity conservation equation with the assumption of no salinity variations along the radial direction is

$$
\frac{\partial S_{s}}{\partial t}+v \frac{1}{r} \frac{\partial S_{s}}{\partial \theta}+w \frac{\partial S_{s}}{\partial z}=\frac{k_{s}}{r^{2}} \frac{\partial^{2} S_{s}}{\partial \theta^{2}}+k_{s}^{v} \frac{\partial^{2} S_{s}}{\partial z^{2}},
$$

where $k_{s}^{v}$ and $k_{s}$ are the vertical and horizontal surface salinity diffusion coefficients. We neglect vertical diffusion between the surface and deep boxes and use the boundary condition $k_{s}^{v} \partial_{z} S_{s}(z=0)=-F_{s}$ at the surface. Vertical advection through the bottom of the annulus is given by the boundary condition $w S(z=H)=-F_{W} S_{s}$ in the polar region, $0<\theta<\pi$, and $w S(z=H)=$ $-F_{W} S_{d}^{S}$ in the midlatitude region, $\pi<\theta<2 \pi$, where $S_{d}^{S}$ is the salinity of the southern deep box that will be introduced later on. This boundary condition is for thermally dominant THC, $Q(t)>0$, where $t$ is time; the case of $Q(t)<0$ is discussed below.

Radial and vertical integration of (5) using the above boundary conditions gives

$$
\begin{aligned}
\frac{\partial S_{s}}{\partial t}+ & \frac{\partial\left(\omega S_{s}\right)}{\partial \theta} \\
= & \frac{k_{s}}{R_{1} R_{2}} \frac{\partial^{2} S_{s}}{\partial \theta^{2}}+\frac{F w(\theta)}{H}\left[S_{s}+\left(S_{d}^{S}-S_{s}\right) \Theta(\theta-\pi)\right] \\
& -F_{s}^{0} \sin \theta .
\end{aligned}
$$

Here, $\Theta(\theta-\pi)$ is the Heaviside function, which enables a concise writing of the exchanges with the deep boxes for both the southern and northern parts of the gyre.

In a similar way, heat conservation leads to an equa- tion for surface temperature $T_{s}$, which is assumed to restore to the atmospheric profile $\bar{T}=T^{*}-T_{0} \sin \theta$, where $T^{*}$ represents the mean atmospheric temperature and $T_{0}$ is the amplitude of the sinusoidal deviation from the mean. The corresponding surface boundary condition is $k_{T}^{v} \partial_{z} T_{s}(z=0)=\Gamma\left(\bar{T}-T_{s}\right)$, where $k_{T}^{v}$ is the vertical thermal diffusion coefficient and $\Gamma$ is the inverse restoring time. The equations for temperature in the surface annulus become

$$
\begin{aligned}
\frac{\partial T_{s}}{\partial t}+ & \frac{\partial\left(\omega T_{s}\right)}{\partial \theta} \\
= & \frac{k_{T}}{R_{1} R_{2}} \frac{\partial^{2} T_{s}}{\partial \theta^{2}}+\frac{F_{W}(\theta)}{H}\left[T_{s}+\left(T_{d}^{s}-T_{s}\right) \Theta(\theta-\pi)\right] \\
& +\Gamma\left(T^{*}-T_{0} \sin \theta-T_{s}\right)
\end{aligned}
$$

where $k_{T}$ is the horizontal thermal diffusion coefficient.

\section{b. Deep boxes}

The deep ocean is represented by a polar box and a midlatitude box. The two boxes have volumes $V_{d}^{N}$ and $V_{d}^{S}$, respectively, and their depth is $D$. Each box is assumed to be completely mixed and is characterized by a single deep temperature $\left(T_{d}^{N}\right.$ and $\left.T_{d}^{S}\right)$ and salinity $\left(S_{d}^{N}\right.$ and $\left.S_{d}^{S}\right)$. The temperature and salinity evolution equations are standard:

$$
\begin{aligned}
V_{d}^{S} \frac{d S_{d}^{S}}{d t} & =Q\left(S_{d}^{N}-S_{d}^{S}\right), \\
V_{d}^{S} \frac{d T_{d}^{S}}{d t} & =Q\left(T_{d}^{N}-T_{d}^{S}\right), \\
V_{d}^{N} \frac{d S_{d}^{N}}{d t} & =-Q \int_{0}^{\pi} f(\theta) S_{s} d \theta-Q S_{d}^{N}, \quad \text { and }
\end{aligned}
$$


TABLE 1. Parameter values used in the model. See the text for their meaning.

\begin{tabular}{ll}
\hline \hline \multicolumn{1}{c}{ Parameter } & \multicolumn{1}{c}{ Value } \\
\hline$H$ & $500 \mathrm{~m}$ \\
$L$ & $3000 \mathrm{~m}$ \\
$R_{1}$ & $500 \mathrm{~km}$ \\
$R_{2}$ & $3000 \mathrm{~km}$ \\
$V_{d}^{S}, V_{d}^{N}$ & $27 \times 10^{7} \mathrm{~km}^{3}$ \\
$\alpha$ & $1.5 \times 10^{-4} \mathrm{~K}^{-1}$ \\
$\beta$ & $7.61 \times 10^{-4} \mathrm{ppt}^{-1}$ \\
$Q_{0}$ & $5 \times 10^{10} \mathrm{~m}^{3} \mathrm{~s}^{-1}$ \\
$\Gamma^{-1}$ & $4 \mathrm{months}$ \\
$T^{*}$ & $16^{\circ} \mathrm{C}$ \\
$T_{0}$ & $10^{\circ} \mathrm{C}$ \\
$k_{s,} k_{T}$ & $10^{3} \mathrm{~m}^{2} \mathrm{~s}^{-1}$ \\
$\tau_{\mathrm{WDC}}=2 \pi / \Omega$ & $1-100 \mathrm{yr}^{-1}$ \\
$F_{s}^{0}$ & $0-25 \mathrm{~m} \mathrm{yr}^{-1}$ \\
\hline
\end{tabular}

$$
V_{d}^{N} \frac{d T_{d}^{N}}{d t}=-Q \int_{0}^{\pi} f(\theta) T_{s} d \theta-Q T_{d}^{N}
$$

Note the exchanges with the downwelling area of the surface gyre, in the form of the above integrals.

\section{c. Thermohaline circulation}

The intensity of the THC, as given by $Q$, is assumed to be proportional to the horizontal pressure gradient as is commonly done in THC box models. A linear equation of state is used, $\rho / \rho_{0}=-\alpha T+\beta S$, with $\alpha$ and $\beta$ being temperature and salinity expansion coefficients and $\rho_{0}$ being a reference density. The densities in the deep polar and midlatitude boxes are $\rho_{d}^{N}$ and $\rho_{d}^{S}$. We also define averaged densities over the sinking and upwelling regions using a weight function that has the same profile as the flux $F_{W}(\theta)$ between the surface annulus and the deep boxes:

$$
\begin{aligned}
& \rho_{s}^{N}=\rho_{0} \int_{0}^{\pi}\left(-\alpha T_{s}+\beta S_{s}\right)|f(\theta)| d \theta \text { and } \\
& \rho_{s}^{S}=\rho_{0} \int_{\pi}^{2 \pi}\left(-\alpha T_{s}+\beta S_{s}\right)|f(\theta)| d \theta .
\end{aligned}
$$

Using these four densities, the THC is defined as in a standard four-box THC model (Tziperman et al. 1994) to be

$$
Q=Q_{0}\left[\rho_{d}^{N}-\rho_{d}^{S}+\delta\left(\rho_{s}^{N}-\rho_{s}^{S}\right)\right],
$$

where $\delta=H / D$.

Note that for the case $Q<0$, which corresponds to a salinity-dominant circulation with water sinking in the equatorial region and upwelling at high latitudes, the system of equations (6), (7), and (8) has to be modified to account for this reverse circulation, using a similar differencing scheme.

The model has been integrated using a leapfrog timestepping scheme with a Robert filter and using a centerdifference formulation in space for the surface-layer

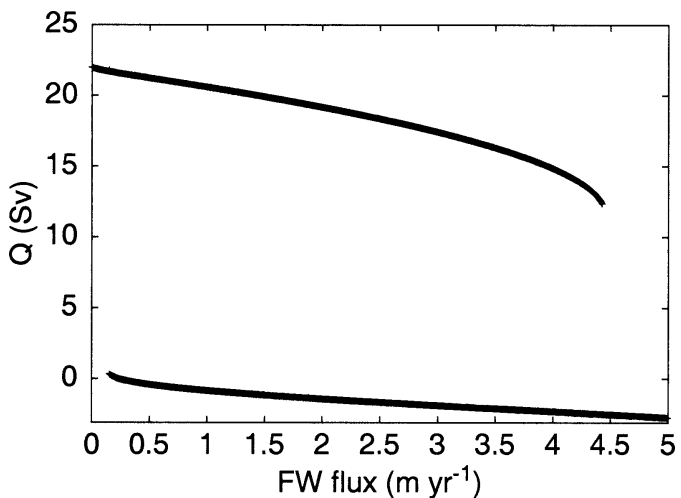

FIG. 3. Intensity of the THC at stable steady-state solutions, as a function of the freshwater forcing amplitude $F_{s}^{0}$ in the absence of a WDC. Positive values refer to thermally dominant THC. Note that, as in the Stommel box model, there is a regime at which there are multiple steady equilibria, both thermally and salinity dominant, for the same FW forcing amplitude.

temperature and salinity equations. The resolution is 512 points along the loop. The minimum time step used for the integration is $6 \mathrm{~h}$. The values of the physical parameters used are reported in Table 1.

\section{Stability behavior with no wind-driven circulation}

When we set the WDC to zero $(\Omega=0)$, the model used here is basically a combination of a box model for the deep ocean and a 1D (in latitude) model for the upper ocean. Because we wish to find out the effects of the WDC on the THC variability, we first need to make sure that we understand the behavior of our model in the absence of the WDC. Figure 3 shows that the multiple equilibria of this model under mixed boundary conditions as a function of the $\mathrm{FW}$ forcing amplitude are pretty much like those of the Stommel's model (Stommel 1961). That is, there are both a thermally dominant and a salinity dominant stable modes for a range of FW forcing, and then for larger FW forcing, only a stable salinity dominant mode remains (Marotzke 1989).

Furthermore, the linearized stability behavior of our model is mostly equivalent to that of the simple four box THC model of Tziperman et al. (1994). The presence of more than just the two boxes of the Stommel model introduces an advection time lag that allows the existence of an oscillatory mode that does not exist in the Stommel two-box model (Kurtze and Restrepo 2001). For small FW fluxes $F_{s}^{0}$, the thermally dominant stable steady state is oscillatory stable. That is, a small perturbation to the steady state leads to damped oscillations that converge back to the steady-state solution. For larger FW fluxes, the oscillatory mode becomes unstable, and a small perturbation leads to growing oscillations and eventually to the salinity-dominated solution (Fig. 4). We will next see that in the presence of 


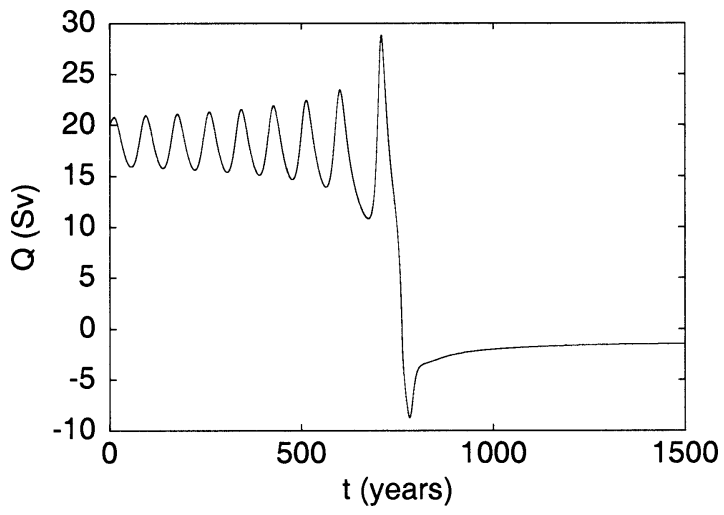

FIG. 4. Transition from the thermally dominated steady state to the salinity-dominated steady state through an unstable oscillatory mode. This time series was obtained by slightly incrementing the FW forcing that led to the thermally dominant steady circulation. The resulting augmented FW amplitude is beyond the regime of stable thermally dominant circulation, hence the switch to a salinity-dominant solution.

the WDC the variability behavior of this model is significantly richer.

\section{Effects of a wind-driven gyre on the thermohaline circulation variability}

We now switch the wind-driven circulation on and define an advection time in the surface layer from the wind-driven circulation to be $\tau_{\mathrm{WDC}}=2 \pi / \Omega$. We then explore the behavior of the model for different intensities of the FW flux at the surface and different time scales of the wind-driven circulation.

\section{a. Stability behavior in the presence of a WDC}

First, fixing $\tau_{\mathrm{WDC}}$ to $70 \mathrm{yr}$, we analyze the solution as function of the FW flux amplitude. In this case, unlike in the case in which the WDC is set to zero, the model allows for self-sustained variability of the THC. Figure 5 shows the bifurcation diagram for increasing FW flux, when the system was initially put in the thermally driven state. The plotted quantity is the value of the THC $Q$ at any local maxima and minima of the time series $Q(t)$ [i.e., we plot the value of $Q(t)$ when $d Q(t) / d t=0$ ]. Crosses indicate steady-state solutions. The plot is made as follows: fix the FW forcing amplitude $F_{s}^{0}$, run the model beyond the initial transients, plot maxima and minima of $Q(t)$, and increase FW by a small amount and repeat.

For small values of the FW flux (below about $F_{s}^{0}=$ $1.7 \mathrm{~m} \mathrm{yr}^{-1}$ ) the system always reaches a steady state. For increased fluxes, the steady-state solution loses stability and a self-sustained periodic variability (limit cycle) is obtained, with period of a few decades to a few hundred years. The limit cycle is seen as two dots in Fig. 5 for a given value of the freshwater forcing amplitude, corresponding to the minimum and maximum

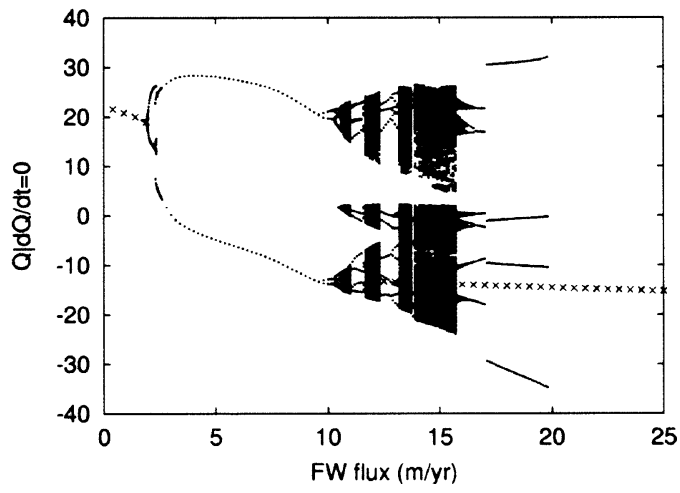

FIG. 5. Bifurcation diagram for the thermohaline variability in the presence of a WDC. Shown are the extrema of the function $Q(t)$ as a function of the surface FW flux amplitude. Crosses indicate steadystate solutions.

of $Q(t)$ during the cycle. For larger-yet FW fluxes, there is a bifurcation that leads to a seemingly chaotic behavior around $F_{s}^{0}=2.3 \mathrm{~m} \mathrm{yr}^{-1}$. For larger fluxes, the oscillations, whose periods have a time scale of a few decades, become larger; for FW flux larger than about $3.1 \mathrm{~m} \mathrm{yr}^{-1}$, the oscillation is between a positive THC and a negative one, corresponding to reversals of the overturning circulation. For (unrealistically) large FW forcing of over $10 \mathrm{~m} \mathrm{yr}^{-1}$, we eventually find a perioddoubling cascade that leads to chaotic solutions and to the typical periodic windows within the chaotic regime. A steady salinity-dominated state is reached only for even larger FW forcing (at about $20 \mathrm{~m} \mathrm{yr}^{-1}$ ). As the freshwater flux is decreased, there is a hysteresis just like that in simple THC meridional box models, so that the salinity-dominant THC is now stable all the way down to $12 \mathrm{~m} \mathrm{yr}^{-1}$, where it becomes unstable again through a Hopf bifurcation.

At this stage, it is already clear that several very interesting and significant things happen to the THC behavior because of the simple addition of a fixed-amplitude WDC. First, there are self-sustained oscillations of the THC rather than only stable and unstable steady states. Second, over a very large range of large-amplitude FW forcing, we obtain large-amplitude THC variability, switching from positive to negative $Q$ during the cycle. This behavior is in contrast to the stable salinity-dominant steady state found in the standard THC models, whether box or continuous 2D (Marotzke et al. 1988).

In Fig. 6 we show the $(S, T)$ phase-space plot for three self-sustained oscillatory solutions: one is a "period one" oscillation, which corresponds to a simple loop in phase space, and is found for FW flux amplitude of $F_{s}^{0}=2 \mathrm{~m} \mathrm{yr}^{-1}$, with a period of $\sim 100 \mathrm{yr}$. The second is a more-complex periodic solution (FW flux $2.35 \mathrm{~m}$ $\mathrm{yr}^{-1}$, period of $\sim 550 \mathrm{yr}$ ), and the third is again a "period one" solution with a larger FW flux (FW flux $2.7 \mathrm{~m}$ $\mathrm{yr}^{-1}$, period of $\sim 50 \mathrm{yr}$ ). The salinity and temperature plotted are the mean values in the sinking region. 


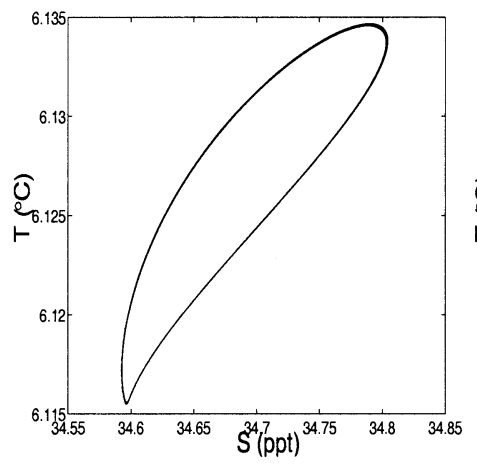

(a)

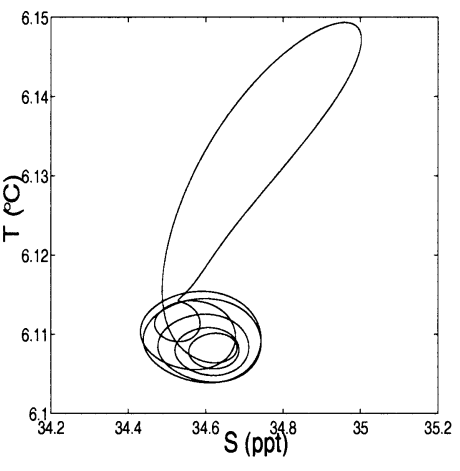

(b)

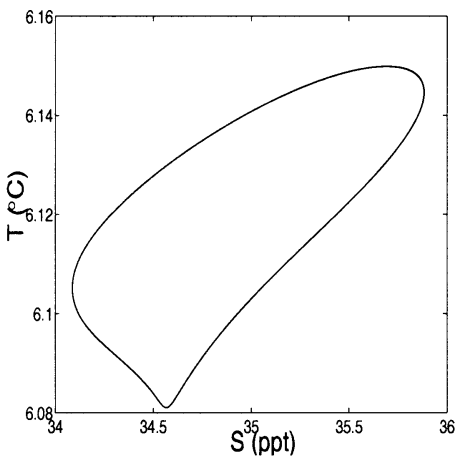

(c)

FIG. 6. Phase-plane plot of $T$ vs $S$ for the northern surface sinking region for $\tau_{\mathrm{WDC}}=70 \mathrm{yr}$ and FW flux of (a) 200 , (b) 235 , and (c) $270 \mathrm{~cm} \mathrm{yr}^{-1}$.

We saw in section 3 that, in the absence of a winddriven gyre, a transition from a thermally dominated to a salinity-dominated steady solution is found when the FW flux is increased (Fig. 3). This transition occurs through an oscillatory unstable solution (Fig. 4). In the presence of a WDC, when the thermally driven steady solution loses stability, the amplitude of the resulting oscillatory solution may saturate and lead to a limit cycle instead of to a switch to a different state. The amplitude of these self-sustained oscillations increases with the FW forcing amplitude, until a switch occurs to larger-amplitude oscillations (Fig. $5, F_{s}^{0} \approx 2.4 \mathrm{~m}$ $\mathrm{yr}^{-1}$ ). There are therefore basically two regimes of selfsustained variability seen in the model: The first is small-amplitude oscillations that correspond to the unstable oscillatory behavior of the model with no WDC and stabilized by the presence of the WDC. The second regime of self-sustained variability is of large-amplitude oscillations that exist for larger-amplitude FW forcing, where the salinity-dominant stable steady state existed in the model with no WDC.

It is important to understand the connection and differences between the stability properties of the THC with and without the WDC. In Fig. 3, with no WDC, there are only stable steady states of two types (thermally dominant and salinity dominant), possibly coexisting in some range of the FW forcing. In the presence of the WDC (Fig. 5), small-amplitude self-sustained oscillations around the thermally dominant state appear at about the amplitude of the FW forcing that would correspond to the change to salinity-dominant steady state in Fig. 3: the thermally dominant stable state loses stability to exponentially growing oscillations, as in the case of no WDC. However, the WDC manages to prevent the oscillations from growing to a large amplitude that would result in a shift to a salinitydominant steady state as occurs in the absence of the WDC. The oscillation's amplitude therefore saturates, and the small-amplitude limit cycle appears. We will see below that the WDC stabilizes the growing oscil- lations by stopping the advective THC instability feedback responsible for the growth of these oscillations (Tziperman et al. 1994). At larger-yet values of the FW forcing, instead of having only a steady-state salinitydominant THC, the presence of the WDC results in large-amplitude oscillations of the THC between thermally dominant and salinity-dominant states.

Note that there exists an FW forcing range for which the time-dependent overturning circulation $Q$ is always positive, the oscillations are relatively small amplitude, and no THC reversal occurs (Fig. 6a). This regime of self-sustained variability may be relevant to present-day climate and is in the same regime of the Chen and Ghil (1995) variability.

\section{b. THC variability regimes as function of both $F W$ forcing and WDC}

Having examined the solution as a function of the FW forcing alone, we now proceed to analyze the selfsustained THC variability as a function of both the FW flux amplitude and the WDC advection time scale $\tau_{\text {WDC }}$. Figure 7 shows the period of the self-sustained variability as a function of these two parameters. For lowamplitude FW flux and small $\tau_{\mathrm{WDC}}$, the system reaches a steady state (regions outside the zero contour line in Fig. 7). When the period of the wind-driven circulation and the FW flux are increased, the solution of the system becomes time dependent. It can be either periodic or chaotic (the latter shown by the shaded regions in the figure).

For a fixed time scale of the wind-driven circulation and a small FW flux (lower zone of Fig. 7), the solution is a thermally dominant steady state. Time-dependent solutions may be found for larger FW forcing, and a (salinity dominated) steady state is eventually reached for even larger $\mathrm{FW}$ fluxes (over $20 \mathrm{~m} \mathrm{yr}^{-1}$ ). As $\tau_{\mathrm{WDC}}$ increases, the salinity-dominated steady state is reached at larger and larger FW fluxes (not shown). As seen in Fig. 5 and briefly explained above, the presence of the 


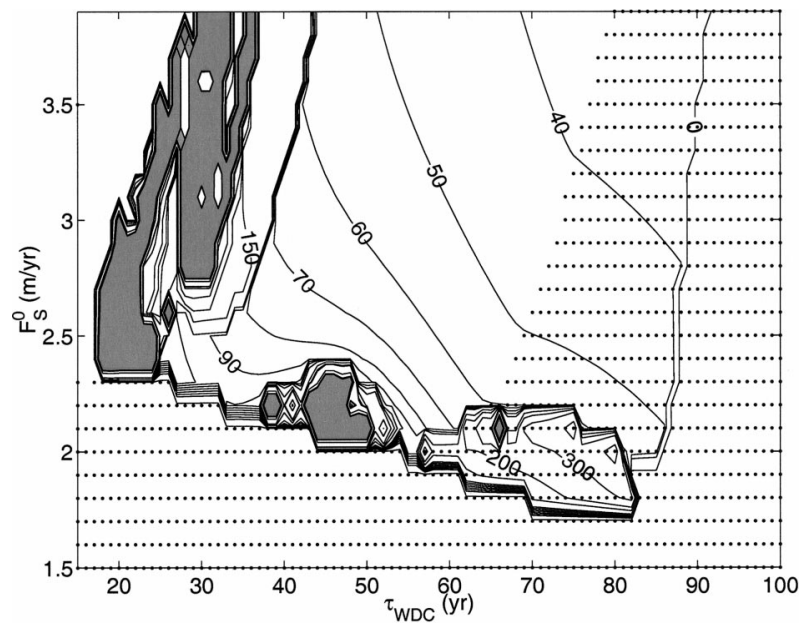

FIG. 7. Isocontours of the period of the thermohaline oscillations (yr) as a function of the freshwater forcing amplitude and the advection time scale of the WDC. The noncontoured region is characterized by a stable steady state with no variability. Shaded regions indicate seemingly chaotic solutions. The dotted region represents the solutions for which the THC $(Q)$ is positive (thermally dominated) throughout the oscillation cycle.

wind-driven circulation allows for the existence of selfsustained (periodic or chaotic) oscillations in the system, and the window of existence of those solutions becomes larger in $F_{s}^{0}$ as the period of the wind-driven circulation grows from about $20 \mathrm{yr}$ to several decades (Fig. 7).

The dotted area in Fig. 7 marks the solutions that correspond to thermally dominated overturning circulation (positive THC $Q$ throughout the oscillation cycle). Not all of these solutions are what could be considered small oscillations about a thermally dominant state, as can be seen in Fig. 5. One class of small-amplitude selfsustained variability is found in the lower-left corner of the contour region of Fig. 7. In areas in parameter regime not marked by dots in the figure, and where oscillatory solutions exist, the overturning circulation changes direction during the oscillations. The period of the periodic self-sustained variability that is plotted in Fig. 7 varies from decades to several hundred years. The case of no wind-driven circulation (see Fig. 3) is recovered for $\tau \rightarrow \infty$, when only steady solutions are possible (thermally and salinity dominated). In this case the advection by the WDC becomes negligible relative to the advection by the THC so that we recover the case of section 3. Note that for very strong intensity of the gyre circulation (small $\tau$ ) no time dependent solutions are found. For (unrealistic) strong wind-driven circulation, the thermally driven circulation loses stability at relatively small freshwater fluxes, and the system sets into the salinity-dominated mode. It seem that when the WDC is sufficiently rapid it simply acts to mix the surface annulus so that it basically functions as a single additional homogeneous box. The model then displays the stability characteristics of a three-box THC box model, which do not include self-sustained oscillations (Rivin and Tziperman 1997).

\section{c. The mechanism of self-sustained THC oscillations in the presence of a WDC}

We now turn our attention to a specific case with interesting variability seen also in Fig. 6b, with $\tau_{\mathrm{WDC}}=$ $70 \mathrm{yr}$ and $F_{s}^{0}$ corresponding to $235 \mathrm{~cm} \mathrm{yr}^{-1}$, to analyze in detail one oscillatory solution of the system. Our objective is to show how self-sustained variability arises from the effects of the WDC. This solution is periodic (Fig. 8), with a period of about $550 \mathrm{yr}$ composed of several faster oscillations of about $80 \mathrm{yr}$ followed by a larger peak in the THC. The THC, as given by $Q$, is always thermally dominated, and the amplitude of the THC oscillations is of the same order as its mean value $\left(Q_{\max } \simeq 2 Q_{\min }\right)$.

Figure 9 shows the surface salinity and temperature anomalies as function of location along the annulus and time during an entire THC oscillation. The shown anomalies are with respect to the time-mean profiles shown in Fig. 10. The mean temperature field in the gyre (Fig. $10 \mathrm{~b}$ ) basically reflects the atmospheric restoring, except for a small dip in the temperature maximum at the southernmost point because of the cold upwelling there. The maximum evaporation occurs at the southernmost point, but the mean salinity profile shows the maximum salinity east of the southernmost point because of the effect of the fresh upwelling water around the southernmost point. The location of the maximum salinity in the surface annulus depends, in fact, on the competing effects of upwelling of freshwater and net evaporation. For weak overturning, air-sea freshwater fluxes dominate and salinity increases as the water flows through the evaporation zone, reaching a maximum at the end of the accumulation zone west of the southernmost point. When the THC is strong, as in the case of Fig. 10 , the large evaporation at $\theta_{S}$ is dominated by the large upwelling of freshwater and the maximum salinity is located in the eastern part of the annulus, where evaporation increases the salinity and yet the upwelling does not yet affect it (Fig. 10a).

Consider now the dynamics of the oscillation shown in Fig. 9, beginning with the small-amplitude oscillations that precede the large peak in $Q(t)$ shown in Fig. 8. These small-amplitude THC oscillations are of the "loop oscillation" type (Welander 1986). This oscillation has been observed in GCMs (Delworth et al. 1993) and has been studied in detail in a four-box model by Griffies and Tziperman (1995), and so we do not need to repeat its mechanism here. In a THC-only model under sufficiently strong FW forcing, the oscillations are also amplified by the "advective" THC instability (Marotzke et al. 1988; Tziperman et al. 1994), which results in a growing amplitude of the oscillations and eventually in a switch to a different steady state (section 3, Fig. 4). With the WDC added here, a reduced THC 

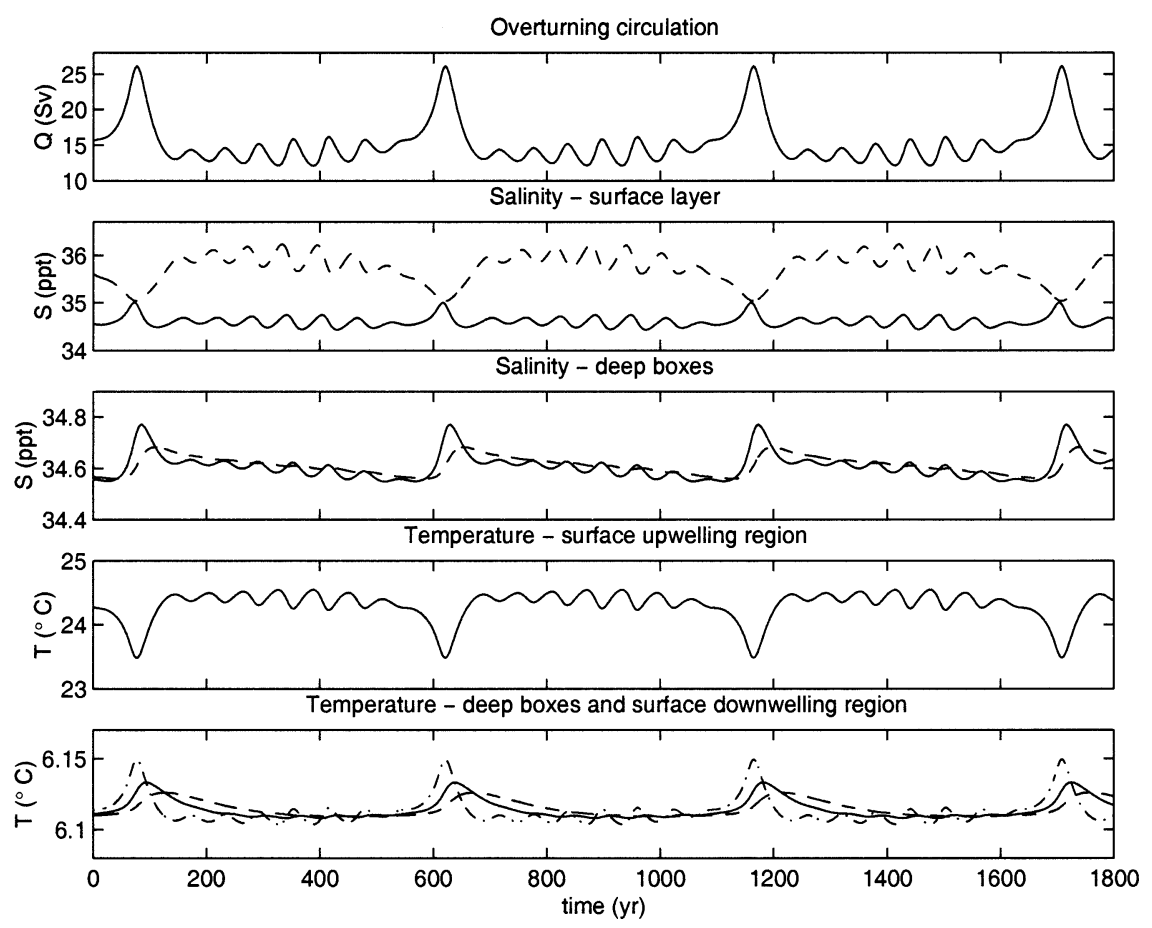

FIG. 8. An example of self-sustained thermohaline oscillations in the presence of a WDC. (top) Thermohaline circulation amplitude (Sv). (second from top) Salinity in the surface layer, averaged over the sinking area (solid line) and over the upwelling region (dashed line). (middle) Salinity in the northern (solid line) and southern (dashed line) deep boxes. (second from bottom) Average temperature in the upwelling surface region. (bottom) Temperature in the northern (solid line) and southern (dashed line) deep boxes, and average temperature in the sinking region (dasheddotted line).

does not necessarily imply a diminished northward salt transport as required by the advective instability mechanism, so that the instability is arrested and self-sustained oscillations arise. Note first that the wind-driven circulation advects salinity anomalies toward the sink- ing area along the western part of the gyre and away from it along the eastern branch. Now, in the presence of a WDC, the salinity anomalies are not symmetric on the east and west branches of the gyre just south of the sinking area. This east-west asymmetry is created in

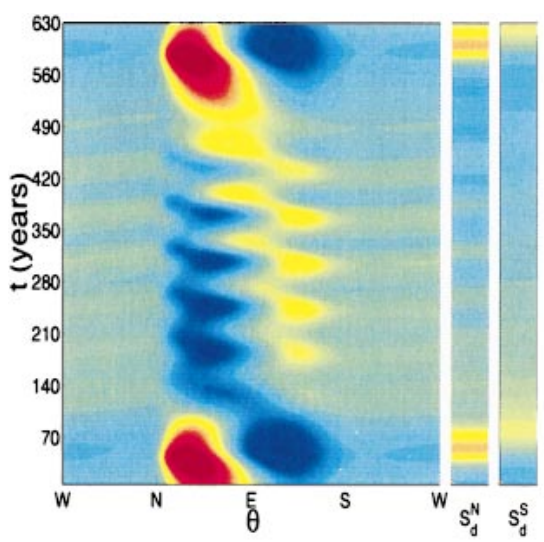

(a)

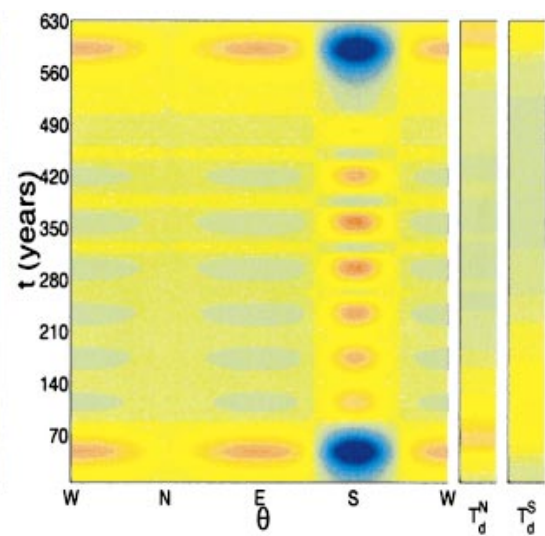

(b)

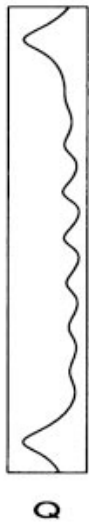

(c)

FIG. 9. (a) Salinity and (b) temperature anomalies during a complete cycle of the oscillation shown in Fig. 8. The larger color panels show the temperature and salinity as functions of time and location along the annulus. The side color bars in (a) and (b) show the variations of the salinity and temperature, respectively, in the deep boxes. (c) The corresponding variation in the intensity of the overturning circulation $Q$. 


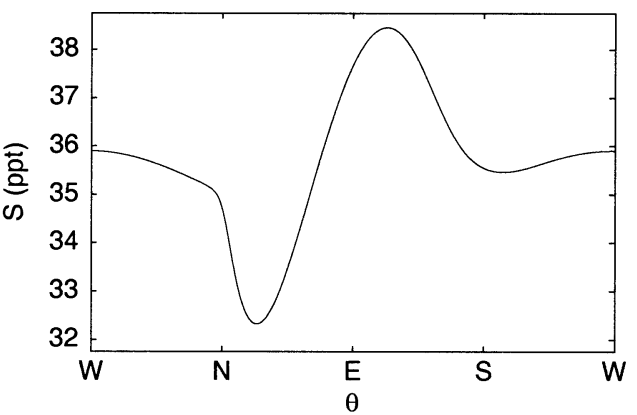

(a)

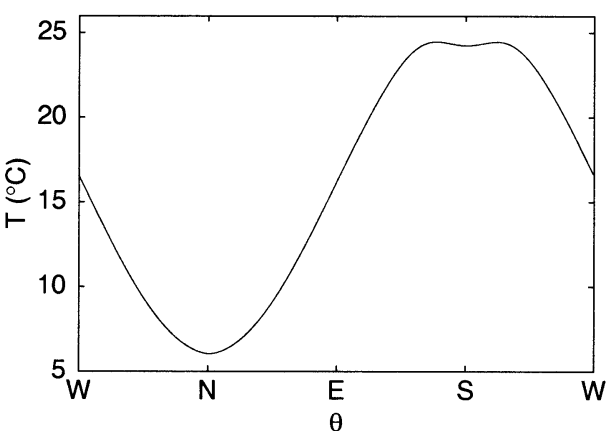

(b)

FIG. 10. Time-mean surface (a) salinity and (b) temperature profiles, averaged over one period of the solution.

part by the nonlinear terms (advection of the perturbation salinity by the perturbation THC). The asymmetry allows the advection by WDC to add or remove salt from the sinking area (depending on the sign of the salinity anomaly in the sinking area) and, by doing that, to arrest the advective feedback and stabilize the oscillations, leading to the limit-cycle solutions observed above.

Next, consider the larger-amplitude peaks of $Q(t)$ seen in Fig. 8 that occur every 500 yr or so. At time $t \simeq 550$ yr in Fig. 9, a large positive salinity anomaly is located in the region of deep-water formation, resulting in a higher density there. This dense surface water increases the strength of the meridional circulation, which correspondingly has a large maximum (Fig. 9c). At the same time, the strong upwelling of cold water from the deep layer in the low-latitude region results in a strong negative temperature anomaly at the surface. This large peak in the THC follows the series of the smaller-amplitude oscillations discussed above. The salinity anomaly resulting in this peak arrives at the sinking area as follows. A positive salinity anomaly forms to the east of the sinking area because of the east-west asymmetry discussed above. As the small oscillations are initially amplified by the advective instability and the THC gets stronger, the advection in the east part of the gyre becomes northward rather than the normal southward advection by the WDC that is observed when the THC is weak. A direct northward advection of the positive salinity anomaly in the eastern branch of the annulus into the sinking region is now possible and results in the larger peaks in the oscillation shown in Fig. 8.

To summarize, the self-sustained variability exists because the advective instability feedback is arrested by the advection of salinity anomalies by the WDC. The time scale of the small-amplitude oscillations is related to the loop mechanism, whereas the occurrence of strong flushes depends on the advection time of a positive salinity anomaly from low latitudes to the north, along the eastern branch of the annulus. In both cases, the oscillation is in the same sense in the $T-S$ phase plane shown in Fig. 6 so that salinity is ahead of the temperature during the entire oscillation, as is typical of THC oscillations (Delworth et al. 1993; Griffies and Tziperman 1995).

\section{d. Gyre versus overturning meridional transports}

The variability analyzed above is especially interesting because it may shed some light on similar variability modes seen in GCM studies. Furthermore, the existence of a WDC in our model allows us a level of comparison with 3D GCM results that was not possible with previous simple meridional THC models (Griffies and Tziperman 1995). To be more specific, the net northward transport in GCMs is often decomposed into "meridional overturning" $([u][X])$ and "gyre" ([u' $\left.\left.X^{\prime}\right]\right)$ components (Bryan 1962) by decomposing each quantity $X$ into a zonal mean $[X]$ and a deviation from that mean $X^{\prime}$, so that

$$
[u X]=[u][X]+\left[u^{\prime} X^{\prime}\right],
$$

where $u$ is the meridional velocity and $X$ is either temperature or salinity. In our simple model, we can use a similar decomposition, in which the zonal mean is simply performed over two points on the annulus that are at the same latitude, in the eastern and western gyre branches. With this decomposition, we see that the anomalous gyre northward transport can be different from zero and can be time dependent even when the wind-driven circulation is not changing (constant $\Omega$ ).

The components of the anomalous northward transport of salt and heat to the sinking region are shown in Fig. 11, computed at a relatively high latitude position $(\theta=3 \pi / 10$ and $\theta=7 \pi / 10)$. The horizontal-gyre anomalous salt transport is out of phase with the overturning circulation, like that seen by the GCM study of Delworth et al. (1993). The phase of the gyre component of heat transport is in phase with the THC component in the northern part of the gyre while in the low latitudes it is out of phase (not shown), again in agreement with GCM studies (Eden and Jung 2001). This result is a very 

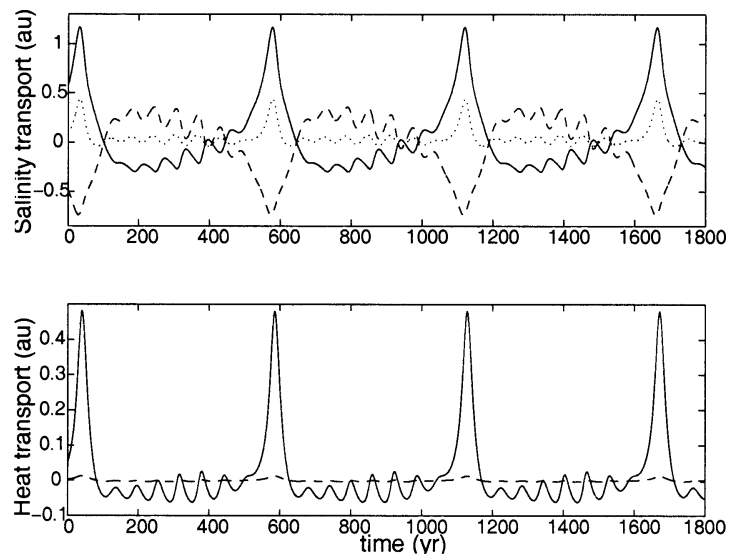

FIG. 11. Zonally averaged northward anomaly (deviation from time-mean value) transport of (top) salt and (bottom) heat. The total anomalous transport (dotted line in the top panel) is decomposed into a gyre contribution (dashed line) and an overturning contribution (solid line). The corresponding overturning circulation is plotted in the top panel of Fig. 8.

encouraging verification that our idealized model is indeed useful for analyzing and understanding more-complex model results.

\section{Conclusions}

Despite the numerous studies on the dynamics of the wind-driven circulation and the thermohaline circulation, there have only been a few works looking at the interaction between the two. In addition, all of these works used GCMs, which are often difficult to analyze in detail or to carry to extreme parameter regimes for a thorough study of variability mechanisms. Our objective here was to formulate the simplest model that may be used to investigate the effect of the WDC on the variability of the THC. We have met this goal by coupling an annulus representing the surface layer of the ocean, in which the WDC is a simple anticyclonic gyre, to two boxes that represent the deep ocean and the THC.

We found several most-interesting new results as a result of adding the WDC to the picture. Unlike simple meridional THC models, which cannot display self-sustained THC variability, our model does show several classes of such variability modes. First, for relatively small FW forcing, we see self-sustained small-amplitude variability that may be relevant to present-day THC variability. Second, at a larger amplitude of the FW forcing that in the absence of a WDC leads to a stable salinity-dominant (reversed) THC, we see large-amplitude THC oscillations that involve spontaneous switches from thermally dominant to salinity-dominant modes, as found, for example, in the GCM study of Weaver et al. (1991). Third, at the boundary between the above two regimes, we find many variability modes of complex character that involve several small-amplitude THC oscillations leading to a periodic large-amplitude THC burst. Last, for large-enough FW forcing, we see a period-doubling cascade that leads to chaotic THC variability. It is very interesting that the presence of even a weak gyre can prevent the model from settling on a stable salinity-dominant THC mode that is invariantly seen in models of the THC alone. This result may strengthen the case of Bice and Marotzke (2001), who found it difficult to make their 3D GCM reproduce a reversed THC mode that has been suggested to exist by some proxy climate evidence (Brass et al. 1982), although note that such a reversed THC mode was found in the GCM study of Zhang et al. (2001). The salinitydominated steady state is stable in our model only for very large FW forcing.

The small-amplitude self-sustained oscillations that are found in the presence of the WDC are one of our main results. We found that there is a simple physical mechanism that explains why such oscillations are possible in the presence of the WDC. The oscillatory mechanism is basically that of the thermohaline loop oscillations (Welander 1986), analyzed in the context of a simple THC box model by Griffies and Tziperman (1995). In such simple meridional THC models under sufficiently strong freshwater forcing, the oscillation grows exponentially in time because of an amplification by the "advective instability feedback" (Marotzke et al. 1988). We have shown here that this instability feedback is arrested by the advection of salinity perturbations by the WDC. This advection leads to a saturation of the amplitude of the oscillations and therefore to the small-amplitude self-sustained oscillations.

The existence of a gyre component in our model allowed us a more-detailed comparison of the model results with 3D GCM results than is possible with idealized meridional THC models (Griffies and Tziperman 1995). We saw, for example, that the decomposition of the meridional fluxes of heat and salt into gyre and meridional-overturning components leads to a most encouraging similarity to some GCM studies (Delworth et al. 1993; Eden and Jung 2001). Such a similarity clearly strengthens the case for using this and similar highly idealized models of the interaction between the WDC and the THC.

Given that our model is extremely idealized, there were obviously many issues that we could not address here. We do not address coupled ocean-atmosphere modes of variability that involve changes to the WDC from the effect of SST variability on the wind stress driving the WDC (Delworth and Greatbatch 2000; Dong and Sutton 2001; Latif 1998; Weaver and Valcke 1998). However, the wealth of results obtained here for thermohaline variability in the presence of a WDC indicates that this area of research is a fertile one that deserves additional studies with a hierarchy of models from the most idealized one used here, through intermediate models, and all the way to coupled GCMs. Such a hierarchical modeling approach may help us to deduce some observable characteristics of coupled THC-WDC 
variability modes that may lead to their verification using available observations.

Acknowledgments. Author CP was partially supported by a scholarship from the Feinberg Graduate School of the Weizmann Institute of Science.

\section{REFERENCES}

Bice, K. L., and J. Marotzke, 2001: Numerical evidence against reversed thermohaline circulation in the warm Paleocene/Eocene ocean. J. Geophys. Res., 106, 11 529-11 542.

Bjerknes, J., 1964: Atlantic air-sea interaction. Advances in Geophysics, Vol. 10, Academic Press, 1-82.

Brass, G. W., J. R. Southam, and W. H. Peterson, 1982: Warm saline bottom water in the ancient ocean. Nature, 296, 620-623.

Bryan, F., 1986: High latitude salinity effects and interhemispheric thermohaline circulation. Nature, 323, 301-304.

Bryan, K., 1962: Measurement of meridional heat transport by ocean currents. J. Geophys. Res., 67, 3403-3414.

Cessi, P., 1994: A simple box model of stochastically forced thermohaline flow. J. Phys. Oceanogr., 24, 1911-1920.

- 1996: Convective adjustment and thermohaline excitability. $J$. Phys. Oceanogr., 26, 481-491.

Chen, F., and M. Ghil, 1995: Interdecadal variability of the thermohaline circulation and high-latitude surface fluxes. J. Phys. Oceanogr., 25, 2547-2568.

Delworth, T., and R. J. Greatbatch, 2000: Multidecadal thermohaline circulation variability driven by atmospheric surface flux forcing. J. Climate, 13, 1481-1495.

_ and M. E. Mann, 2000: Observed and simulated multi decadal variability in the Northern Hemisphere. Climate Dyn., 16, 661676.

_ , S. Manabe, and R. J. Stouffer, 1993: Interdecadal variations of the thermohaline circulation in a coupled ocean-atmosphere model. J. Climate, 6, 1993-2011.

Dickson, R. R., J. Lazier, J. Meincke, P. Rhines, and J. Swift, 1996: Long-term coordinated changes in the convective activity of the North Atlantic. Progress in Oceanography, Vol. 38, Pergamon, 241-295.

Dijkstra, H. A., 2000: Nonlinear Physical Oceanography. Kluwer Academic, $480 \mathrm{pp}$.

Dong, B. W., and R. T. Sutton, 2001: The dominant mechanism of variability in Atlantic Ocean heat transport in a coupled oceanatmosphere GCM. Geophys. Res. Lett., 28, 2445-2448.

Eden, C., and T. Jung, 2001: North Atlantic interdecadal variability: Oceanic response to the North Atlantic Oscillation. J. Climate, 14, 676-691

Frankignoul, C., P. Muller, and E. Zorita, 1997: A simple model of the decadal response of the ocean to stochastic wind forcing. $J$. Phys. Oceanogr., 27, 1533-1546.

Griffies, S. M., and E. Tziperman, 1995: A linear thermohaline oscillator driven by stochastic atmospheric forcing. J. Climate, $\mathbf{8}$, $2440-2453$.

Hall, A., and R. J. Stouffer, 2001: An abrupt climate event in a coupled ocean-atmosphere simulation without external forcing. Nature, 409, 171-174.

Kurtze, D. A., and J. M. Restrepo, 2001: Advective time lags in box models. J. Phys. Oceanogr., 31, 1828-1842.

Kushnir, Y., 1994: Interdecadal variations in North Atlantic sea sur- face temperature and associated atmospheric conditions. J. Climate, 7, 141-157.

Latif, M., 1998: Dynamics of interdecadal variability in coupled ocean-atmosphere models. J. Climate, 11, 602-624.

, and T. P. Barnett, 1994: Causes of decadal climate variability over the North Pacific and North America. Science, 266, 634637.

_ , and _ 1996: Decadal variability over the North Pacific and North America: Dynamics and predictability. J. Climate, 9, $2407-2423$.

Manabe, S., and R. J. Stouffer, 1999: The role of the thermohaline circulation in climate. Tellus, 51, 91-109.

Marotzke, J., 1989: Instability and multiple equilibria of the thermohaline circulation. Oceanic Circulation Models: Combining Data and Dynamics, D. L. T. Anderson and J. Willebrand, Eds., Kluwer, 501-511.

_ P. Welander, and J. Willebrand, 1988: Instability and multiple steady states in a meridional-plane model of the thermohaline circulation. Tellus, 40A, 162-172.

Marshall, J., H. Johnson, and J. Goodman, 2001: A study of the interaction of the North Atlantic Oscillation with ocean circulation. J. Climate, 14, 1399-1421.

Moron, V., R. Vautard, and M. Ghil, 1998: Trends, interdecadal and interannual oscillations in global sea surface temperatures. $\mathrm{Cli}$ mate Dyn., 14, 545-569.

Pierce, D. W., T. P. Barnett, and U. Mikolajewicz, 1995: Competing roles of heat and freshwater flux in forcing thermohaline oscillations. J. Phys. Oceanogr., 25, 2046-2064.

Rivin, I., and E. Tziperman, 1997: Linear versus self-sustained interdecadal thermohaline variability in a coupled box model. $J$. Phys. Oceanogr., 27, 1216-1232.

Stommel, H., 1961: Thermohaline convection with two stable regimes of flow. Tellus, 13, 224-230.

Tziperman, E., J. R. Toggweiler, Y. Feliks, and K. Bryan, 1994 Instability of the thermohaline circulation with respect to mixed boundary conditions: Is it really a problem for realistic models? J. Phys. Oceanogr., 24, 217-232.

Weaver, A. J., and T. M. C. Hughes, 1994: Rapid interglacial climate fluctuations driven by North Atlantic Ocean circulation. Nature, 367, 447-450.

— , and E. S. Sarachik, 1991: The role of mixed boundary conditions in numerical models of the ocean's climate. J. Phys. Oceanogr., 21, 1470-1493

- and S. Valcke, 1998: On the variability of the thermohaline circulation in the GFDL coupled model. J. Climate, 11, 759767.

_ E. S. Sarachik, and J. Marotzke, 1991: Freshwater flux forcing of decadal and interdecadal oceanic variability. Nature, $\mathbf{3 5 3}$ 836-838.

Welander, P., 1982: A simple heat-salt oscillator. Dyn. Atmos. Oceans, 6, 233-242.

_ 1986: Thermohaline effects in the ocean circulation and related simple models. Large-Scale Transport Processes in the Oceans and Atmosphere, D. L. T. Anderson and J. Willebrand, Eds., NATO ASI Series, Reidel, 163-200.

Winton, M., and E. S. Sarachik, 1993: Thermohaline oscillation induced by strong steady salinity forcing of ocean general circulation models. J. Phys. Oceanogr., 23, 1389-1410.

Yin, F., and E. S. Sarachik, 1995: Interdecadal thermohaline oscillations in a sector ocean general circulation model: Advective and convective processes. J. Phys. Oceanogr., 25, 2465-2484.

Zhang, R., M. J. Follows, J. P. Grotzinger, and J. Marshall, 2001: Could the Late Permian deep ocean have been anoxic? Paleoceanography, 16, 317-329. 